\title{
SASL: Saliency-Adaptive Sparsity Learning for Neural Network Acceleration
}

\author{
Jun Shi, Jianfeng Xu, Kazuyuki Tasaka, Zhibo Chen, Senior Member, IEEE
}

\begin{abstract}
Accelerating the inference of CNNs is critical to their deployment in real-world applications. Among all pruning approaches, the methods of implementing a sparsity learning framework have shown effectiveness as they learn and prune the models in an end-to-end data-driven manner. However, these works impose the same sparsity regularization on all filters indiscriminately, which can hardly result in an optimal structuresparse network. In this paper, we propose a Saliency-Adaptive Sparsity Learning (SASL) approach for further optimization. A novel and effective estimation of each filter, i.e., saliency, is designed, which is measured from two aspects: the importance for prediction performance and the consumed computational resources. During sparsity learning, the regularization strength is adjusted according to the saliency, so our optimized format can better preserve the prediction performance while zeroing out more computation-heavy filters. The calculation for saliency introduces minimum overhead to the training process, which means our SASL is very efficient. During the pruning phase, in order to optimize the proposed data-dependent criterion, a hard sample mining strategy is utilized, which shows higher effectiveness and efficiency. Extensive experiments demonstrate the superior performance of our method. Notably, on ILSVRC2012 dataset, our approach can reduce $49.7 \%$ FLOPs of ResNet50 with very negligible $0.39 \%$ top- 1 and $0.05 \%$ top-5 accuracy degradation.
\end{abstract}

Index Terms-convolutional neural network (CNN), sparsity learning, adaptive, acceleration, compression.

\section{INTRODUCTION}

Convolutional neural networks (CNNs) have been widely applied in a variety of computer vision applications, including classification [1]-[3], object detection [4], [5] and semantic segmentation [6], [7], etc. Scaling up the size of models has been the main reason for the success of deep learning. For instance, the depth of ImageNet Classification Challenge [8] winner models, has evolved from 8 of AlexNet [1] to over 100 of ResNet [3]. Empirically, larger networks can exhibit better performances, while they are also known to be heavily over-parameterized [9].

However, large CNNs might be incompatible with their deployment in real-world applications, suffering severely from the massive computational and storage overhead. Therefore, it is really necessary to obtain the compact networks with efficient inference.

Jun Shi and Zhibo Chen are with the CAS Key Laboratory of Technology in Geo-spatial Information Processing and Application System, University of Science and Technology of China, Hefei 230027, China. Jianfeng $\mathrm{Xu}$ and Kazuyuki Tasaka are with the KDDI Research, Japan (e-mail: shi1995@mail.ustc.edu.cn; ji-xu@kddi-research.jp; ka-tasaka@kddiresearch.jp; chenzhibo@ustc.edu.cn). This work was supported in part by NSFC under Grant U1908209, 61632001 and the National Key Research and Development Program of China 2018AAA0101400.
Pruning [10] is a common approach to slim neural networks via removing the redundant weights, filters and layers. Weight pruning can achieve a higher compression ratio, but will lead to unstructured sparsity of CNNs, which makes it hard to leverage the efficient Basic Linear Algebra Subprograms (BLAS) libraries [11]. Therefore, structured sparsity pruning becomes more attractive since it can reduce the model size as well as accelerate the inference.

Among all structured sparsity pruning approaches, sparsity learning (SL) [12]-[30], or called sparsity regularization, is a popular and powerful direction these days. These works introduce sparsity regularization on structures during the training phase. Training with structured regularization can transfer significant features to a small number of filters and automatically obtains a structure-sparse model [13]. However, in these SL approaches, the sparsity regularization imposed on all filters is the same, without the consideration of specified characteristics of different parts of the models. Theoretically and empirically, two main results can be incurred from such indiscriminate regularization. First, the performance of important filters for prediction is also influenced by the equal regularization, so the final prediction precision after SL may drop by a large margin. And sometimes it is difficult to restore the performance after pruning. Second, different filters consume different computational resources. According to our analysis and experimental observations, traditional SL tends to zero out lots of light filters while retaining the computation-heavy filters. Therefore, we can hardly obtain the optimal structure-sparse networks using the indiscriminate SL.

In this paper, we propose a novel SL form, namely SaliencyAdaptive Sparsity Learning (SASL), to learn better compact neural networks. The saliency of a convolutional filter is considered from two aspects: 1) the importance for prediction performance, which is defined as the change in the loss function induced by removing this filter from the neural network. 2) the consumed resources, especially the computational costs. During SL, the regularization for every filter will be adjusted adaptively according to the saliency of filters, and the calculation for saliency will only lead to very minimum overhead. In the pruning phase, saliency is also proven to be a better criterion than other methods, and the proposed hard sample mining strategy can further improve the effectiveness and efficiency of saliency. In brief, the main contributions of this paper are summarized as follows.

- We propose a novel form of sparsity learning, the Saliency-Adaptive Sparsity Learning (SASL). Compared with traditional SL, our optimized format can better preserve the performance of models and reduce more 
computation for inference without too much overhead.

- In the pruning stage, we observe and analyze that saliency is a better criterion than previous methods. Since saliency is data-dependent, a hard sample mining strategy is proposed to further enhance the effectiveness and efficiency of it.

- Extensive experiments on two benchmark datasets for various CNN architectures demonstrate the effectiveness and efficiency of the proposed approach. Typically, on ILSVRC-2012 dataset, 49.7\% FLOPs of ResNet-50 are reduced with only $0.05 \%$ top- 5 accuracy degradation, which significantly outperforms other state-of-theart methods.

The remainder of this paper is organized as follows. we review works related to network pruning and sparsity learning in Section II Section III introduces the motivation of this paper and the details of the proposed SASL approach. The experimental results and corresponding analyses are presented in Section IV. Finally, we conclude our work in Section V. The code of SASL will be shared on our website: http://staff. ustc.edu.cn $/ \sim$ chenzhibo/resources.html for public research.

\section{RELATED WORKS}

Network pruning has been a long-studied project ever since the very early stage of neural network. In this section, we review the significant development of network pruning.

Weight Pruning. In the 1990s, Optimal Brain Damage [31] and Optimal Brain Surgeon [32] were proposed, in which, unimportant weights were removed based on the Hessian of the loss function. In recent work, Han et al. [10] brought back this idea by pruning the weights of which the absolute values are smaller than a pre-set threshold. And Molchanov et al. [33] proposed Variational Dropout to prune redundant weights. Moreover, [34] formulated weight pruning into an optimization problem by finding the weights to minimize the loss while satisfying the pruning cost condition. Kang [35] proposed a weight pruning scheme considering the accelerator. Frankel et al. [36] developed the lottery ticket theory, based on which, only using the weights of winning tickets can present comparable or even better performance than the original model. But finding the winning tickets initialization is complex and computational expensive. To solve this, Morcos et al. [37] proposed a generalization method which allows reusing the same wining tickets across various datasets. And Ding et al. [38] focused on the optimizer and designed a novel momentum-SGD, which shows superior capability to find better winning tickets. However, the nature of unstructuredsparsity makes it only yield effective compression but cannot speed up the inference without dedicated hardware/library.

Structured Pruning. Therefore, much attention has been focused on structured pruning to accelerate the inference of neural networks. Filter pruning, or called channel pruning, is the most common and flexible way of structured pruning, since filter is the smallest unit of structure.

Many heuristic methods are proposed to prune filters based on the handcrafted features. For example, based on the smaller-norm-less-important belief, Li et al. [39] proposed pruning filters according to the filter weight norm. Average percentage of zeros (APoZ) in the output was used in [40] to measure the importance of filters. He et al. [41] pruned filters to minimize the feature reconstruction error of the next layer. Similarly, [11] pruned redundant filters via estimating the statistics of its next layer. Yu et al. [42] implemented feature ranking to obtain importance score and propagated it throughout the network to find the pruned filters. And Chin et al. [43] considered pruning as a ranking problem and then compensated the layer-wise approximation error to improve the performance of previous heuristic metrics. [44] used reinforcement learning to decide the pruned ratio of each layer. $\mathrm{He}$ et al. [45] proposed the concept of soft pruning, which allows pruned filters to recover during training. Furthermore, Huang et al. [46] trained pruning agents to remove structures in a data-driven way. [47] used Taylor-expansion to estimate the importance of each filter and then iteratively pruned the least important filters. He et al. [48] proposed pruning the redundant filters via geometric median. Zhao et al. [49] proposed a variational Bayesian pruning scheme based on the distribution of channel saliency. Liu et al. [50] proposed the concept of MetaPruning, which combined meta learning with evolutionary algorithm to provide an efficient automatic channel pruning approach. These methods directly pruned filters of the unsparsified models, which would erroneously abandon useful features and result in huge accuracy degradation.

Sparsity Learning. So recent approaches [12]-[30] have adopted sparsity learning to introduce structured sparsity in the training phase. Zhang et al. [12] incorporated sparsity constraints into the loss function to reduce the number of filters. Similarly, Wen et al. [13] utilized Group Lasso to automatically obtain structured sparsity during training. Moreover, Liu et al. [14] proposed network slimming to apply $\ell_{1}$-norm on the channel scaling factors, which can reuse the $\gamma$ parameters in Batch-Normalization layers so there is little training overhead. After SL, filters with small scaling factors will be pruned. And [19] extended this idea, which utilized the scaling factors for coarser structures apart from filters, such as layers and branches. Lin et al. [28] further used generative adversarial learning to pick the structures for pruning. Morphnet [30] proposed iteratively shrinking and expanding a network to automate the neural network design. In the shrinking procedure, the satisfying regularization is imposed on activations to distinguish the necessary filters. In [26], a neuron-budget-aware sparsity learning method is proposed to avoid trial-and-error. Compared with directly pruning, these methods obtain structure-sparse neural networks in the training stage. As a consequence, redundant filters could be removed with less accuracy decline. However, the above methods impose the same sparsity regularization on all filters indiscriminately, which is the critical problem to be solved in this paper. Most recently, several works also recognized this problem. Yang et al. [29] proposed the method of DeepHoyer, which made use of the tool of Hoyer to replace the $\ell_{1}$ regularizer and achieved high performance. Yun et al. [22] tried to tackle this with the proposed approach of Trimmed- $\ell_{1}$ regularizer, which allows the filters with largest norm penaltyfree from regularization. But this optimization method is still 
quite simple, and as discussed later, Trimmed $\ell_{1}$ regularizer can be seen as a special case of our work.

\section{Proposed ApproACH}

\section{A. Motivation}

We start by showing and analyzing the flaws of previous indiscriminate sparsity learning. Typically, we can formulate the optimization objective of sparsity learning as:

$$
\text { Loss }=f(\mathcal{W}, \mathcal{D})+\lambda \cdot \sum_{i=1}^{S} R\left(w_{i}\right) .
$$

Here $\mathcal{W}$ is the overall trainable weights of current $\mathrm{CNN}$, while $\mathcal{D}=\left(x_{i}, y_{i}\right)_{i=1}^{N}$ is the used training set. $f(\mathcal{W}, \mathcal{D})$ is the original loss function of the task, such as cross entropy. And $R\left(w_{i}\right)$ is the sparsity regularization for filter $w_{i}$. Generally, the form of $R(\cdot)$ can be Group Lasso or $\ell_{1}$-norm penalty on the structure-corresponding scaling factors, which is widely used to achieve sparsity. $\lambda$ is the hyper-parameter to balance the original loss and the sparsity regularization. Note that all the filters $w$ share the same parameter $\lambda$ indiscriminately.

This is a coarse format of sparsity learning that may lead to critical problems. The sparsity is achieved without guidance, and we will show the drawbacks from two aspects in the following sections III-A1 and III-A2, Actually, we can integrate some prior information into sparsity learning, thus better structure-sparse neural networks can be obtained.

1) Importance of Filters: Different filters in a neural network are of different importance. The same as [47], in this paper we define the importance $\mathcal{I}_{m}$ of filter $w_{m}$ as the error induced by the removal of it. Under an i.i.d. assumption, this error can be measured as the squared difference of prediction losses with and without filter $w_{m}$ :

$$
\mathcal{I}_{m}=\left(E(\mathcal{D}, \mathcal{W})-E\left(\mathcal{D}, \mathcal{W} \mid w_{m}=0\right)\right)^{2} .
$$

For a given model $\mathcal{M}$, we denote the corresponding optimal pruned model as $\mathcal{M}^{\prime}$. And in the transition from $\mathcal{M}$ to $\mathcal{M}^{\prime}$, the probability of filter $w_{m}$ to be pruned is denoted as $p_{m}$. Intuitively, the less important a filter is, the more likely it is to be pruned. So we can assume that the relationship between $\mathcal{I}_{m}$ and $p_{m}$ conforms to an inverse correlation function, as Fig. 1 shows.

Here we simply divide all filters of a model into three categories. For the least important filters, pruning them directly is not risky. On the contrary, the most significant filters are essential for the prediction precision, and any impact on them will incur a performance decline of the model. The role of sparsity learning is to help identify the filters in the middle part, i.e., $w_{2}$ in Fig. 1

One of the critical issues of indiscriminate sparsity learning is that the importance difference of filters is disregarded. Regularization on the important filters can lead to massive accuracy drop and sometimes this drop cannot be recovered. Moreover, regularization effects on $w_{3}$ deteriorate the representational capacity of current model, so the sparsity learning would fail to maximally identify the redundant filters.

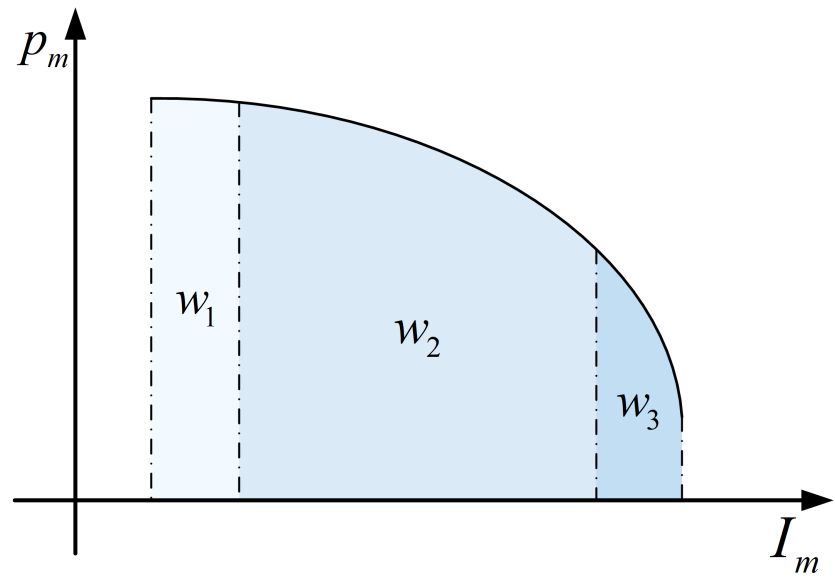

Fig. 1. Simplified relationship between importance and pruning possibility of filter $m$. Filters that are important are not likely to be pruned. Deep color denotes high importance. Best viewed in color.

2) Computational Resources: Most of the previous approaches equate the goal of network pruning, i.e., reducing more consumed resources, with removing more structures, such as convolutional filters. However, without the consideration of consumed computational resources (or memory footprint) of different filters, the directions of these two statements are different, and sometimes the gap cannot be ignored. Specifically, convolutional filters of different layers in one model would cost different resources. Basically, it depends on the following three factors: 1. input feature map size; 2 . number of input feature maps; 3 . filter size. Without guidance, traditional sparsity learning such as $\ell_{1}$-norm scheme can only zero out more filters rather than reducing more computational complexity.
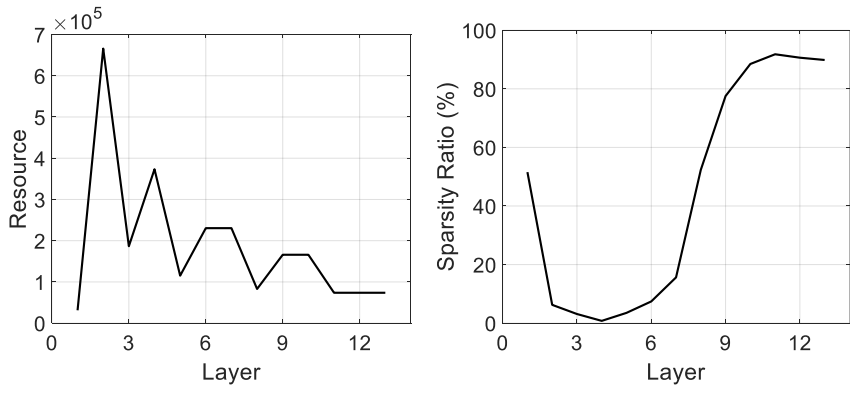

Fig. 2. Normalized consumed resources and sparsity ratio of filters in different layers of VGG-Net. This strong inconsistency arises from the indiscriminate sparsity learning.

Here we conduct an experiment to show the inconsistency of the indiscriminate sparsity learning. We implement network slimming [14] for VGG-Net on CIFAR-10 dataset [51]. When planning to prune $70 \%$ filters, we record the distribution of filters to be pruned which possess the smallest scaling factors. All these scaling factors are less than $10^{-2}$, so we can assume that these filters have been already sparsified. Then we display the normalized computational complexity and sparsity ratio of filters in all layers in Fig. 2.

We can observe that traditional sparsity learning algorithm 
tends to zero out more light filters, while most of the computation-heavy filters, such as filters in layer $2 \sim 6$, are retained. Obviously, indiscriminate sparsity learning cannot obtain the optimal structure-sparse networks in terms of complexity reduction.

\section{B. Saliency Estimation}

Therefore, in order to solve the critical problems incurred from traditional sparsity learning, we propose imposing adaptive regularization on different filters discriminatively. A new attribute: saliency, is introduced to estimate filters and then guide the regularization distribution, which is considered from two aspects: the importance for final prediction and the consumed computational resources.

First we need to estimate the importance $\mathcal{I}$ of all filters during sparsity learning. The most precise evaluation for importance is as Eq. (2) shows. However, this is extremely computationally expensive, since it requires evaluating all kinds of versions of the neural network, one for each pruned filter. A method to avoid this is to approximate $\mathcal{I}_{m}$ in the vicinity of $\mathcal{W}$ using second-order Taylor expansion as Optimal Brain Damage [31] shows:

$$
\mathcal{I}_{m}^{(2)}(\mathcal{W})=\left(g_{m}^{T} \cdot w_{m}-\frac{1}{2} w_{m} \cdot H_{m}^{T} \cdot \mathcal{W}\right)^{2} .
$$

Here $g_{m}=\frac{\partial f(\mathcal{W}, \mathcal{D})}{\partial w_{m}}$ and $H_{i, j}=\frac{\partial^{2} f(\mathcal{W}, \mathcal{D})}{\partial w_{i} \partial w_{j}}$ are the gradient and Hessian of filter $w_{m}$, respectively. However, computing Hessian matrices sometimes is also computationally expensive, especially for large networks. So we can adopt a more compact approximation, i.e., using the first-order expansion as [47] does. So the importance can be calculated as:

$$
\mathcal{I}_{m}^{(1)}(\mathcal{W})=\left(g_{m}^{T} \cdot w_{m}\right)^{2} .
$$

In this format, calculating the importance will not bring too much computation overhead since $g_{m}$ is already known from the back-propagation during training.

Then we need to estimate the consumed computational resources $\mathcal{R}$ of different filters. We denote the three influential factors: input feature map size (considering padding pattern and stride), number of input feature maps and filter size as $\mathcal{S}_{f m}, \mathcal{N}_{f m}$ and $\mathcal{S}_{f}$, respectively. So the normalized computational resources $\mathcal{R}_{m}$ of filter $m$ can be calculated as:

$$
\mathcal{R}_{m}=\mathcal{S}_{f m} \cdot \mathcal{N}_{f m} \cdot \mathcal{S}_{f} .
$$

Note that the calculation for $\mathcal{N}_{f m}$ should be dynamic in both sparsity learning and pruning, since the number of valid feature maps can be decreased. During sparsity learning, the sparsified filters are excluded, of which the scaling factors are smaller than $10^{-2}$. And in the pruning phase, the pruned filters will not be counted in.

Finally, we can calculate the saliency $\mathcal{S} \mathcal{A} \mathcal{L}_{m}$ of filter $m$ as:

$$
\mathcal{S} \mathcal{A} \mathcal{L}_{m}=\frac{\mathcal{I}_{m}}{\mathcal{R}_{m}}=\frac{\left(g_{m}^{T} \cdot w_{m}\right)^{2}}{\mathcal{S}_{f m} \cdot \mathcal{N}_{f m} \cdot \mathcal{S}_{f}} .
$$

In this definition, saliency can be understood as the average prediction gain with a unit computational cost. We will show in the following sections that saliency is very effective in both sparsity learning and pruning.

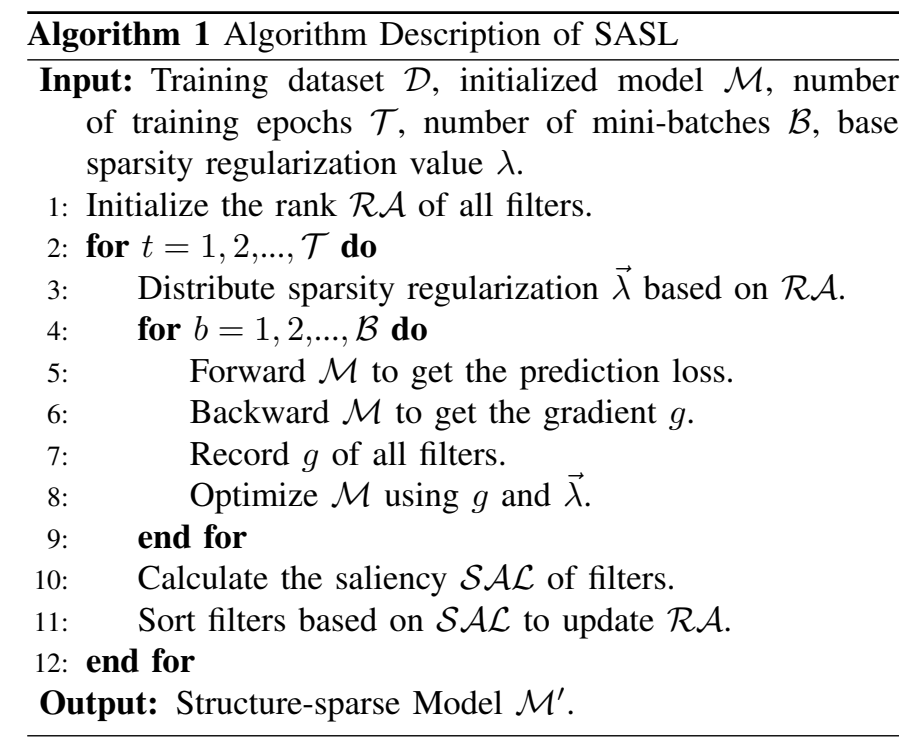

\section{Adaptive Sparsity Regularization}

Based on saliency estimation, we can adaptively set the regularization strength according to this feature of all filters. The indiscriminate format of Eq. (1) now becomes as:

$$
\text { Loss }=f(\mathcal{W}, \mathcal{D})+\sum_{i=1}^{\mathcal{S}} \lambda_{i} \cdot \mathcal{R}\left(w_{i}\right) .
$$

Note that the value of regularization factor $\lambda_{i}$ is now dependent on the filter $w_{i}$. In this paper, we implement this idea based on the scaling factor scheme as network slimming [14] does, but it can be easily generalized to all kinds of sparsity learnings. In the scaling factor scheme, for each filter (including the one in convolutional layers and fully-connected layers), a scaling factor is introduced, which is multiplied by the output of the corresponding filter. Then during sparsity learning, the regularization term (i.e., $R(\cdot)$ in Eq. (7) is imposed on these scaling factors. These scaling factors can be seen as the agents to identify the filters.

Since Batch-Normalization (BN) layer has been widely adopted by most of the modern CNNs, we can reuse the $\gamma$ parameters in $\mathrm{BN}$ as the scaling factors. Typically, BN layer performs the following transformation in the network:

$$
\hat{z}=\frac{z_{\text {in }}-\mu_{\mathcal{B}}}{\sqrt{\sigma_{\mathcal{B}}^{2}+\varepsilon}} ; \quad z_{\text {out }}=\gamma \hat{z}+\beta .
$$

Here $z_{\text {in }}$ and $z_{\text {out }}$ are the input feature and output feature of the BN layer, while $\mu_{\mathcal{B}}$ and $\sigma_{\mathcal{B}}$ are the values of mean and standard deviation of input features among current batch $\mathcal{B}$. $\gamma$ and $\beta$ are the trainable affine transformation parameters, i.e., scale and shift. Therefore, we can directly leverage the $\gamma$ parameters in $\mathrm{BN}$ as the scaling factors since they perform the same function. In this way, we would not introduce any additional parameters.

Due to the distinct saliency distributions of different models, directly using the norm of saliency to guide the regularization distribution is not generalized enough. So we propose utilizing saliency to sort all filters, and then based on the ranking of filters, a hierarchy scheme is adopted to adaptively set the 
sparsity regularization. Typically, for the filters of the most significant class, no regularization will be imposed on, and for the least significant filters, we distribute the strongest regularization penalty. Dedicated design for the hierarchy classification and norm of regularization can lead to better results, but it also requires time-consuming manual working. Therefore, we simply adopt a five-class hierarchy scheme. The filters are classified uniformly according to the saliency score ranking, and the corresponding regularization multiplying factors are set as $\{0 \times, 1 \times, 2 \times, 3 \times, 4 \times\}$. We will show in the experiments that even such simple design can lead to an excellent result.

Note that the saliency estimation and ranking are all along with the sparsity learning, which means the significant filters at the beginning might be rated as useless during the training. Therefore, the regularization distribution is always dynamic. It can precisely observe the current state and adopt the appropriate action. The detailed algorithm of SASL is summarized in Algorithm 1

\section{Iterative Pruning with Hard Sample Mining}

After sparsity learning, an effective criterion is needed to discard filters. Most of the previous sparsity learning approaches, such as [14], prune filters from the energy term, i.e., norm of the scaling factor, due to the "smaller-norm-lessimportant" belief. However, this criterion is not an excellent one since energy cannot fully represent the importance (see in Eq. (4), importance also takes gradient into consideration) and the consumed resource is not even considered. So we propose using saliency as the criterion for pruning, several advantages of which are listed as follows:

1) Saliency is effective to estimate filters, from both the importance and resource aspects.

2) It is globally consistent throughout the whole network and sensitivity analysis for each layer is not needed.

3) This method is able to be applied to any layer in the network, including traditional convolutions, skip connections and fully-connections. It is worth mentioning that pruning skip connections may lead to inconsistency between the feature maps of residual and identity branches. For this situation, we generate the fake all-zero feature maps for the output of each branch if necessary. In this way, the element-wise addition process in skip connections can be done as usual.

4) Although saliency estimates the fine-grained structure, i.e., filter, using saliency to prune can also automatically remove the coarse structures, such as res-block or multibranch, if the whole structure is thought to be redundant.

The overall proposed pruning procedures are illustrated in Fig. 3 Compared with single pass pruning, an iterative pruning and fine-tuning strategy is adopted to achieve better results, since the estimation for importance and resource is always changing during the pruning process. As a data-dependent metric, saliency is sensitive to the used input data. And one main drawback is the potentially intensive computation because all training data are utilized for saliency estimation during pruning, especially when the training set is huge, such as ILSVRC-2012. And this is much more obvious for our multi-pass pruning scheme. However, we do not need to make use of the full training set for saliency estimation. Inspired by OHEM [52], in this paper, we propose a hard sample mining approach for optimization. In detail, before pruning, we calculate the training loss of each sample. Then we pick the samples with top $30 \%$ losses, which are defined as the hard samples. And in the pruning phase, we only use the hard samples for saliency estimation. The extra computation of saliency estimation can be dramatically reduced while the pruning effect can be even better than the original scheme which uses the whole training set. We will analyze this in the experiment part.

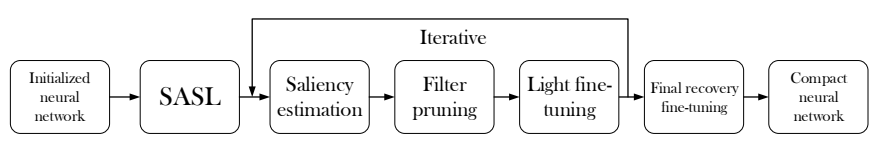

Fig. 3. Proposed iterative pruning framework. We adopt an iterative pruning and fine-tuning strategy, which guarantees precise saliency estimation.

\section{EXPERIMENTAL RESULTS AND ANALYSES}

In this section, we empirically demonstrate the effectiveness of SASL on two benchmark datasets. We implement our method based on the publicly available deep learning framework PyTorch [53]. We introduce the used datasets and pruned neural networks in $\mathrm{IV}-\mathrm{A}$ and then present the training details in IV-B. In IV-C and IV-D, we show the experimental results on the two datasets. The complexity analysis of SASL is presented in IV-E We implement a series of ablation experiments in IV-F to further reveal the superiority of the proposed framework. The running time acceleration analysis is shown in IV-G. Finally, we present several discussions in IV-H to better analyze the special designs.

\section{A. Datasets and Network Models}

1) Datasets: Two classical classification datasets: CIFAR10 [51] and ILSVRC-2012 [8], are adopted in this paper. CIFAR-10 dataset consists of images with resolution $32 \times 32$, which is classified into 10 classes. The training and test sets contain 50,000 and 10,000 images, respectively. A standard data augmentation scheme [54], [55], including shifting and mirroring, is used. All input data is normalized with channel means and standard deviations.

As for ILSVRC-2012, it is a huge dataset with 1.2 million training images and 50,000 validation images which are drawn from 1,000 classes. We adopt the same data augmentation scheme as PyTorch official examples [53] for training. In the test stage, we will report the single-center-crop validation error of the model as the prediction performance.

2) Network Models: On CIFAR-10 dataset, we evaluate our framework on two popular network architectures: VGGNet [56] and ResNet [3]. VGG-Net is originally designed for ILSVRC-2012 classification task. In our experiment, a 
TABLE I

RESUlts OF PRUNING ON CIFAR-10 DATASET.

\begin{tabular}{|c|c|c|c|c|c|c|}
\hline & Approach & $\begin{array}{c}\text { Baseline } \\
\text { Accuracy }(\%)\end{array}$ & $\begin{array}{c}\text { Pruned } \\
\text { Accuracy }(\%)\end{array}$ & Accuracy $\downarrow(\%)$ & Params $\downarrow(\%)$ & FLOPs $\downarrow(\%)$ \\
\hline \multirow{6}{*}{ VGG-Net } & L1 |39| & 93.25 & 93.40 & -0.15 & 64.0 & 34.2 \\
\hline & SSS [19| & 93.96 & 93.63 & 0.33 & 66.7 & 36.3 \\
\hline & FPGM [48] & 93.58 & 93.54 & 0.04 & - & 34.2 \\
\hline & GAL |28| & 93.96 & 93.42 & 0.54 & 82.2 & 45.2 \\
\hline & VCP [49] & 93.25 & 93.18 & 0.07 & 73.3 & 39.1 \\
\hline & SASL & 93.69 & 93.89 & -0.20 & 86.9 & 49.5 \\
\hline \multirow{9}{*}{ Res-56 } & L1 |39| & 93.04 & 93.06 & -0.02 & 13.7 & 27.6 \\
\hline & SFP |45| & 93.59 & 92.26 & 1.33 & - & 52.6 \\
\hline & $\mathrm{CP}|41|$ & 92.80 & 91.80 & 1.00 & - & 50.0 \\
\hline & NISP [42] & - & - & 0.03 & 42.6 & 43.6 \\
\hline & FPGM [48] & 93.59 & 93.49 & 0.10 & - & 52.6 \\
\hline & GAL $28 \mid$ & 93.26 & 93.38 & -0.12 & 11.8 & 37.6 \\
\hline & VCP [49] & 93.04 & 92.26 & 0.78 & 20.5 & 20.3 \\
\hline & SASL & 93.63 & 93.88 & -0.25 & 18.9 & 35.9 \\
\hline & SASL* & 93.63 & 93.58 & 0.05 & 36.6 & 57.1 \\
\hline \multirow{8}{*}{ Res-110 } & L1 [39] & 93.53 & 93.30 & 0.23 & 32.4 & 38.6 \\
\hline & SFP |45] & 93.68 & 93.38 & 0.30 & - & 40.8 \\
\hline & NISP [42] & - & - & 0.18 & 43.8 & 43.3 \\
\hline & FPGM [48] & 93.68 & 93.74 & -0.06 & - & 52.3 \\
\hline & GAL |28| & 93.50 & 93.59 & -0.09 & 4.1 & 18.7 \\
\hline & VCP [49] & 93.21 & 92.96 & 0.25 & 41.3 & 36.4 \\
\hline & SASL & 93.83 & 93.99 & -0.16 & 31.9 & 51.7 \\
\hline & SASL* & 93.83 & 93.80 & 0.03 & 54.3 & 70.2 \\
\hline
\end{tabular}

SASL and SASL* are the conservative and aggressive schemes, respectively. Accuracy $\downarrow$ is the prediction performance drop between pruned model and baseline model, the smaller, the better. A negative value of Accuracy $\downarrow$ means performance improvement after pruning.

variation of VGG-Net for CIFAR-10 dataset is taken from [39]. For ResNet, two ResNets of 56 layers and 110 layers are used. On ILSVRC-2012 dataset, we adopt the deep ResNet-50 for pruning. Batch-Normalization layers are adopted in all models to achieve better performance.

\section{B. Training Details}

1) Normal Training: In normal training, we train all the CNNs from scratch as baselines. All the models are trained using the optimizer of stochastic gradient descent (SGD). On CIFAR-10 dataset, we train VGG-Net and ResNet using minibatch size of 64 for 160 and 240 epochs, respectively. The initial learning rate is set as 0.1 , and is divided by 10 at $50 \%$ and $75 \%$ of the total number of training epochs. And on ILSVRC-2012 dataset, we train ResNet-50 for 90 epochs, with a batch size of 256 . The initial learning rate is 0.1 , and we divide it by 10 after 30 and 60 epochs. A weight decay of $10^{-4}$ and a Nesterov momentum [57] of 0.9 without dampening are used in our experiments to improve the training performance. We also adopt the weight initialization introduced by [58].

2) Sparsity Learning and Pruning: Although our framework can adaptively distribute the sparsity regularization, a base regularization value $\lambda$ should be determined in advance, which can control a trade-off between prediction performance and structure sparsity. Empirically, we use relative larger $\lambda$ for the simple VGG-Net $\left(10^{-4}\right)$, while smaller $\lambda$ for the complicated ResNet $\left(3 \times 10^{-5}\right)$. Other settings are the same as normal training.

In our experiments, the pruning procedure is achieved via building a new compact model and then copying the retained weights from the original model. And saliency is used as the metric to help discard the specified filters from the structuresparse models. Suppose the number of filters to be discarded is $\mathcal{N}$. In every iteration, we will prune $5 \% * \mathcal{N}$ filters with the least saliency scores. Therefore, 20 pruning iterations are needed. During experiments, we find that the saliency ranking 
TABLE II

Pruning ResNet-50 ON ILSVRC-2012 DatASET.

\begin{tabular}{lccccccc}
\hline \multirow{2}{*}{ Approach } & $\begin{array}{c}\text { Baseline Top-1 } \\
\text { Acc. }(\%)\end{array}$ & $\begin{array}{c}\text { Pruned Top-1 } \\
\text { Acc. }(\%)\end{array}$ & $\begin{array}{c}\text { Baseline Top-5 } \\
\text { Acc. }(\%)\end{array}$ & $\begin{array}{c}\text { Pruned Top-5 } \\
\text { Acc. }(\%)\end{array}$ & $\begin{array}{c}\text { Top-1 } \\
\text { Acc. } \downarrow \text { (\%) }\end{array}$ & $\begin{array}{c}\text { Top-5 } \\
\text { Acc. } \downarrow(\%)\end{array}$ & $\begin{array}{c}\text { FLOPs } \downarrow \\
(\%)\end{array}$ \\
\hline SFP [45| & 76.15 & 74.61 & 92.87 & 92.06 & 1.54 & 0.81 & 41.8 \\
CP [41] & - & - & 92.20 & 90.80 & - & 1.40 & 50.0 \\
GDP [59| & 75.13 & 71.89 & 92.30 & 90.71 & 3.24 & 1.59 & 51.3 \\
DCP [60| & 76.01 & 74.95 & 92.93 & 92.32 & 1.06 & 0.61 & 55.8 \\
ThiNet [1]| & 72.88 & 71.01 & 91.14 & 90.02 & 1.87 & 1.12 & 55.8 \\
SSS [19] & 76.12 & 74.18 & 92.86 & 91.91 & 1.94 & 0.95 & 31.3 \\
GAL [28] & 76.15 & 71.80 & 92.87 & 90.82 & 4.35 & 2.05 & 55.0 \\
Taylor-FO [47] & 76.18 & 74.50 & - & - & 1.68 & - & 45.0 \\
FPGM [48] & 76.15 & 75.59 & 92.87 & 92.63 & 0.56 & 0.24 & 42.2 \\
C-SGD [61] & 75.33 & 74.93 & 92.56 & 92.27 & 0.40 & 0.29 & 46.2 \\
HRank [62] & 76.15 & 74.98 & 92.87 & 92.33 & 1.17 & 0.54 & 43.8 \\
SASL & $\mathbf{7 6 . 1 5}$ & $\mathbf{7 5 . 7 6}$ & $\mathbf{9 2 . 8 7}$ & $\mathbf{9 2 . 8 2}$ & $\mathbf{0 . 3 9}$ & $\mathbf{0 . 0 5}$ & $\mathbf{4 9 . 7}$ \\
SASL* & $\mathbf{7 6 . 1 5}$ & $\mathbf{7 5 . 1 5}$ & $\mathbf{9 2 . 8 7}$ & $\mathbf{9 2 . 4 7}$ & $\mathbf{1 . 0 0}$ & $\mathbf{0 . 4 0}$ & $\mathbf{5 6 . 1}$ \\
\hline
\end{tabular}

SASL and SASL* are the conservative and aggressive schemes, respectively. Acc. $\downarrow$ is the prediction performance drop between pruned model and baseline model, the smaller, the better.

of filters does not change obviously in the early pruning stage. So we also develop a fast pruning scheme, in which we prune $20 \% * \mathcal{N}$ filters at the beginning 3 iterations. In this way, we only need 11 iterations and the final performance is almost the same.

3) Fine-Tuning: Using pruning, we can obtain more compact models. Then we need to fine-tune them to restore the performance. The learning rate of fine-tuning for all models is set as $10^{-4}$. On CIFAR-10 datasets, we fine-tune the pruned models for 20 epochs, while on ILSVRC-2012 dataset, we only fine-tune the pruned ResNet for 10 epochs.

\section{Results on CIFAR-10}

For the CIFAR-10 dataset, we test our SASL on VGGNet, ResNet-56 and ResNet-110. As shown in TABLE I, our SASL outperforms other state-of-the-art methods in all three networks. For VGG-Net, SASL reduces $49.5 \%$ FLOPs with even $0.2 \%$ accuracy improvement, while previous works [19], [28], [39], [48], [49] are worse in both two aspects. For example, GAL [28] only prunes $45.2 \%$ FLOPs and incurs $0.54 \%$ accuracy degradation.

For ResNet-56 and ResNet-110, we prune filters of different ratios to achieve different trade-offs between accuracy and complexity. In TABLE I. SASL means the conservative scheme that tries to preserve the accuracy, while SASL* denotes the aggressive scheme. During sparsity learning, the aggressive scheme slightly increases the base regularization value, and prunes relatively more filters in the pruning stage. Comparing with other works, we can find that our framework also achieves state-of-the-art performance for ResNet. For pruning ResNet-56, SASL* reduces more FLOPs than FPGM [48] (57.1\% v.s. 52.6\%) and better preserves the accuracy (degradation: $0.05 \%$ v.s. $0.10 \%$ ). On ResNet-110, SASL achieves a higher FLOPs reduction (51.7\% v.s. 36.4\%) with $0.16 \%$ accuracy increase, while VCP [49] harms the performance of prediction. These results demonstrate the effectiveness of SASL, which strongly aligns with our previous analyses.

\section{Results on ILVSRC-2012}

SASL is also evaluated on ILSVRC-2012 dataset for pruning ResNet-50. Similarly, we adopt both the conservative and aggressive schemes. TABLE [II shows the superior performance of SASL. Under various pruned FLOPs ratios, our approach consistently achieves state-of-the-art performance when compared with other methods [11], [19], [28], [41], [45], [47], [48], [59]-[62]. To be specific, the conservative scheme SASL reduces $49.7 \%$ FLOPs with very negligible $0.39 \%$ top- 1 and $0.05 \%$ top-5 accuracy degradation, while SSS [19] incurs huger performance deterioration (1.94\% top- 1 and $0.95 \%$ top5 accuracy drops) and only prunes $31.3 \%$ FLOPs. Our SASL* also performs well, which reduces more FLOPs than DCP [60] ( $56.1 \%$ v.s. $55.8 \%$ ) with better performance maintaining. Compared with previous methods, SASL estimates the saliency of different filters and intelligently distributes the regularization to obtain better structure-sparse networks, which is the main cause of its superior performance.

\section{E. Computational Complexity Analysis of SASL}

Here we analyze the extra complexity of our SASL in addition to the original training procedure. The introduced computational complexity arises from three parts: 1 . saliency estimation for every batch; 2 . saliency aggregation and ranking for every epoch; 3 . hierarchy regularization distribution 
TABLE III

COMPUTATIONAL COMPLEXITY ANALYSIS FOR ONE EPOCH.

\begin{tabular}{llll}
\hline Action & Complexity & Frequency & FLOPs \\
\hline SE & $O(n)$ & 782 & $4.7 \times 10^{6}$ \\
SA\&R & $O(n \log n)$ & 1 & $\leq 2.1 \times 10^{6}$ \\
RD & $O(n)$ & 1 & $2.0 \times 10^{3}$ \\
Summation & - & - & $6.8 \times 10^{6}$ \\
\hline Inference & - & - & $6.3 \times 10^{12}$ \\
\hline
\end{tabular}

SE, SA\&R and RD refer to saliency estimation, saliency aggregation $\&$ ranking and regularization distribution, respectively. Inference complexity considers all 50,000 samples in CIFAR-10.

for every epoch. Actually, since the necessary variables for saliency estimation can be directly obtained from the training procedure, the computational complexity of these three processes is really negligible. We use ResNet-56 on CIFAR10 as an example, which possesses 2,032 filters (including nodes in fully-connected layers). The detailed complexity for one training epoch has been shown in TABLE III] It is worth mentioning that the FLOPs value is approximated, in which the comparing operation is also counted.

From TABLE III] we can find that the extra computational complexity is really small, far less than $1 \%$ of the inference complexity of ResNet-56, let alone the complexity of other processes of training, such as back-propagation and parameter updating. Therefore, our SASL brings minimal overhead to the overall training procedure.

\section{F. Ablation Study}

In this part, we conduct a series of ablation experiments to validate the effectiveness of proposed schemes. For simplicity and reliability, all the following experiments are conducted on CIFAR-10 for ResNet-56. Without specification, the hyperparameter setting is the same as stated in IV-B

1) Different Sparsity Regularization: First, we analyze the effectiveness of the special saliency-adaptive sparsity learning. For comparison, we run the experiment of traditional indiscriminate sparsity learning as baseline. To better show the insight, we also replace the regularization guider, i.e., saliency, with two of its factors, importance and resource. After all kinds of sparsity learnings, we prune filters of different ratios of the models to get a close complexity reduction. Then we fine-tune all the models and show the classification accuracy fluctuation in TABLE IV] In this table, we can see that SASL works much better than the indiscriminate one, with $0.47 \%$ accuracy improvement. The importance- and resource-guided versions can also improve the performance than baseline, but they are both worse than the integrated version. Saliency gives attention to both aspects so it can better guide the sparsity learning.

2) Hierarchy Scheme Extension: Based on the saliency, we classified the filters into five classes and then adaptively impose the regularization. Here we change the hierarchy scheme with different number of classes to explore the influence of this parameter. One-class scheme equals the traditional sparsity
TABLE IV

RESULTS OF DIFFERENT REGULARIZATIONS FOR SPARSITY LEARNING.

\begin{tabular}{l|cccc}
\hline & Tradition & Importance & Resource & Saliency \\
\hline FLOPs $\downarrow$ & $57.0 \%$ & $57.0 \%$ & $57.1 \%$ & $57.1 \%$ \\
ACC $\downarrow$ & $0.52 \%$ & $0.36 \%$ & $0.33 \%$ & $0.05 \%$ \\
\hline
\end{tabular}

learning and five-class scheme is the proposed one. We also change the base regularization value according to the number of classes so as to impose the same amount of regularization. The regularization multiplier $r_{k}$ for class number of $k$ is set to satisfy the following equation:

$$
r_{k} \cdot \sum_{i=0}^{k-1} i \cdot \frac{1}{k} \cdot r_{\text {base }}=\sum_{i=0}^{4} i \cdot \frac{1}{5} \cdot r_{\text {base }}
$$

Here $r_{\text {base }}$ is regularization value for the scheme of five classes. The results after pruning and fine-tuning are shown in Fig. 4 We can find that with the increase of classes, there is a growing in final accuracy, and the increase is slow down when the number of classes is already large. In order to be more generalized for all models and avoid being too complicated, we adopt the scheme of five classes at last. Note that finetuning this parameter for the specific neural network may even lead to better results.

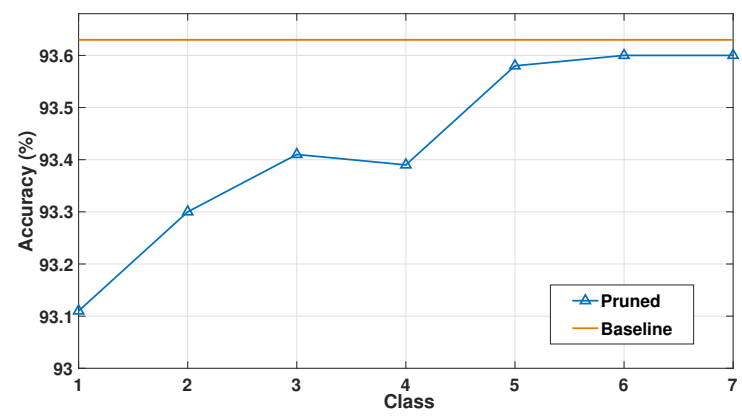

Fig. 4. Results of different settings for number of classes. With the increase of classes, the final accuracy is improved.

We can observe that there is a performance drop at class $=4$. Here we present a possible explanation. Generally, for the scheme with more classes, unimportant filters will be imposed on larger regularization, while this is not always the case. To be specific, in our experiment, the base regularization multiplier of class $=4$ is a little bit smaller than class = 3 (refer to Eq. (9)). As a consequence, there is an overlap in the regularization strength on some filters between these two schemes. In other word, at class $=4$, some unimportant filters will be imposed on smaller regularization, and some important filters will be imposed on larger regularization, given that both schemes provide the same amount of regularization. These special cases could lead to performance drop. With the number of classes going up, the overlapping areas are gradually eliminated (since the classification for filters is more fine-grained) and the distribution for regularization is more rational, so the general trend of performance is rising up. 
3) Saliency as Criterion: After sparsity learning, we need to adopt a criterion to discard filters. In this paper, we claim that saliency is also an excellent metric, which takes both the importance and resource term into consideration. Here we compare saliency with other criteria for pruning to show the superiority. The most common criterion in previous sparsity learning approaches is based on the energy term, i.e., the norm of scaling factors or mean value of filters. We also prune the filters from the aspects of importance and resource. The difference between saliency and resource is that the importance factor in saliency (in Eq. (6)) is replaced with the energy term. TABLE V shows the accuracy results of reducing the same ratio of FLOPs. Not surprisingly, saliency is better than other criteria.

TABLE V

RESUlts of DifFERENT CRITERIA FOR PRUNING.

\begin{tabular}{lcccc}
\hline & Energy & Importance & Resource & Saliency \\
\hline FLOPs $\downarrow$ & $57.0 \%$ & $56.9 \%$ & $57.2 \%$ & $57.1 \%$ \\
ACC $\downarrow$ & $0.25 \%$ & $0.22 \%$ & $0.18 \%$ & $0.05 \%$ \\
\hline
\end{tabular}

4) Input Data for Saliency Estimation: The proposed criterion for pruning, i.e., saliency, is data-dependent, which means saliency estimation could be sensitive to the used input data. Directly using all training data would bring huge complexity overhead, especially for the multi-pass pruning scheme. In this paper, we propose a hard sample mining strategy for efficient and effective saliency estimation. We compare it with using all the training data for saliency estimation to prune filters. Surprisingly, hard sample mining strategy can not only reduce the complexity overhead for the saliency estimation, but also improve the overall performance $(93.58 \%$ v.s. $93.51 \%)$. We attribute the success to the correlation between hard samples and test sets. Easy samples cannot provide too much information for guidance, and sometimes such information can be deemed as the noise, which would influence the accurate pruning action. Preserving the performance for hard samples can make the model work better on the test set.

5) Staircase Regularization Distribution: In our approach, we use a staircase hard multiplier $\{0 \times, 1 \times, 2 \times, 3 \times, 4 \times\}$ to adjust the regularization, rather than directly applying saliency to construct the multiplier. This is due to the following two reasons: 1 . the range of saliency scores is quite large; 2 . models always differ from each other on the distribution of saliency scores. For example, the saliency scores of filters (aggregated for one epoch) in normally trained ResNet-56 range from $1.46 \times 10^{-10}$ to $3.20 \times 10^{-3}$, while it is from $4.38 \times 10^{-18}$ to $1.47 \times 10^{-2}$ for VGG-Net. As a consequence, it is not convenient to directly apply the value of saliency as the guider.

Here we show the experiment results of using saliency as the multiplier. For the filters with large saliency scores, they are supposed to be important, so we should decrease the regularization strength. Therefore, the reciprocal of saliency, denoted as $R S$, can be an effective guider. In the first trial, we directly use $R S$ as the multiplier and conduct an experiment. Not surprisingly, the neural network cannot be trained since the overall loss function is overwhelmed by the regularization, considering that the max value of $R S$ is so large. The cross-entropy loss cannot provide any effective guide for the training. Therefore, we multiply $R S$ with $10^{-7}$ (based on a grid search) to alleviate the strength of it and conduct another experiment. In this trial, the final accuracy of the pruned model reaches $92.59 \%$, lower than our designed staircase scheme (accuracy $=93.58 \%$ ). We can see that directly using saliency to guide the regularization makes it inconvenient and timeconsuming to control the regularization amount, and also leads to sharp feedback effect on the filters, thus the performance is deteriorated.

\section{G. Running Time Reduction}

In addition to the metric of FLOPs reduction, we also measure the running time of pruned models and original models to show the effect of realistic acceleration. TABLE VI shows the results, including VGG-Net on CIFAR-10 and ResNet-50 on ILSVRC-2012. We can observe that there is a gap between the FLOPs reduction rate and running time reduction rate, which may be due to the limitation of IO delay and buffer switch. Also, the acceleration performance of the plain network, i.e., VGG-net, is much better, considering the fact that ResNet is much more complicated. The skipconnections will occupy more time for data flow and transmission. Note that the running time acceleration requires no special libraries/hardware.

TABLE VI

COMPARISON ON FLOPS AND RUNNING TIME REDUCTION.

\begin{tabular}{llcc}
\hline Model & Scheme & FLOPs $\downarrow(\%)$ & Running Time $\downarrow(\%)$ \\
\hline VGG-Net & SASL & 49.5 & 46.2 \\
\hline \multirow{2}{*}{ ResNet-50 } & SASL & 49.7 & 37.8 \\
& SASL $*$ & 56.1 & 41.8 \\
\hline
\end{tabular}

VGG-Net is on CIFAR-10 dataset, while ResNet-50 is on ILSVRC-2012 dataset.

\section{H. Discussions}

Based on the experimental results and comparisons with other approaches, here we present several discussions to better analyze our special designs.

1) CE Loss as Compensation for Regularization: As we point out earlier, in traditional indiscriminate sparsity learning, the regularization term, i.e., $R(\cdot)$ in Eq. (1), is without guidance, while the original objective function $f(\mathcal{W}, \mathcal{D})$, such as cross entropy (CE) loss in classification task, can compensate the regularization effect to some extent. The $\mathrm{CE}$ losss gradient will weaken the effect of the regularizer if the filter is truly important to the final performance. However, this compensation effect is still weak, due to the non-convex optimization procedure of current deep learning framework from back-propagation. As previous method [14] shows, one way to avoid removing important filters is to reduce the 
regularization strength. The drawback of this method is that it requires a multi-pass "SL-pruning" iteration to obtain enough sparisified filters, which is very inefficient and computationally expensive. In comparison, our design makes use of more prior information to provide a hierarchy scheme, which allows larger regularization. Therefore, efficient single-pass SL while preserving the prediction performance is possible.

In addition, the above guidance from CE loss only considers the importance term. In our work, consumed resources term is also integrated into the estimation metric (saliency), which can better guide the sparsity direction for FLOPs reduction.

2) Performance Improvement after Pruning: As we can observe in TABLE I], several pruned models achieves higher prediction precision after SASL. For example, when reducing $35.9 \%$ FLOPs of ResNet-56 on CIFAR-10, we can improve the performance with $0.25 \%$ (from $93.63 \%$ to $93.88 \%$ ). We hypothesize this is due to the regularization effect of sparsity learning, which naturally selects important features in intermediate layers of a neural network. This effect can remove the redundancy as well as the noisy information. This phenomenon is obvious on the simple task such as CIFAR-10 classification, while for pruning networks on the complex ILSVRC-2012 dataset, the performance improvement is not very evident since the redundancy of original models is much smaller.

3) Comparison with Trimmed- $\ell_{1}$ : The most recent work [22] also recognized the critical problem of traditional indiscriminate sparsity learning and proposed the method of Trimmed- $\ell_{1}$ regularizer, in which, filters with the largest norm will not be imposed with regularization, and [22] only implemented this on the simple MNIST dataset for LeNet-5 [63].

Compared with Trimmed- $\ell_{1}$, our work optimizes sparsity learning and works better in two aspects. First, as pointed out earlier, the main belief smaller-norm-less-important of [22] works not very well, so the distinguishing of filters only based on the norm cannot be very precise. In comparison, our proposed metric, i.e., saliency, is integrated with the Taylorexpansion approximated importance and consumed computational resources, which can better represent the significance of filters. Second, [22] only leaves several filters to be penaltyfree, which can be viewed as the two-class hierarchy scheme, a special case of our work. As seen in Fig. 4, the simple twoclass scheme cannot help search the optimal structure-sparse networks effectively.

\section{CONCLUSION}

Current deep convolutional neural networks are effective with high inference complexity. In this paper, we first analyze the critical problems of previous indiscriminate sparsity learning approach and then propose a novel structured regularization form, namely SASL, which can distribute the regularization value for each filter according to saliency adaptively. SASL can better preserve the performance and zeroout more computation-heavy filters. We also propose using saliency as the criterion for pruning. To further improve the effectiveness and efficiency of this data-dependent criterion, we utilize a hard sample mining strategy, which shows better performance and also saves computational overhead. Experiments demonstrate the superiority of SASL over other stateof-the-art methods. In future work, we plan to investigate how to combine SASL with other acceleration algorithms that are orthogonal to our scheme, such as matrix decomposition, to obtain better performance.

\section{REFERENCES}

[1] A. Krizhevsky, I. Sutskever, and G. E. Hinton, "Imagenet classification with deep convolutional neural networks," in Advances in neural information processing systems, 2012, pp. 1097-1105.

[2] C. Szegedy, W. Liu, Y. Jia, P. Sermanet, S. Reed, D. Anguelov, D. Erhan, V. Vanhoucke, and A. Rabinovich, "Going deeper with convolutions," in Proceedings of the IEEE conference on computer vision and pattern recognition, 2015, pp. 1-9.

[3] K. He, X. Zhang, S. Ren, and J. Sun, "Deep residual learning for image recognition," in Proceedings of the IEEE conference on computer vision and pattern recognition, 2016, pp. 770-778.

[4] J. Dai, Y. Li, K. He, and J. Sun, "R-FCN: Object detection via regionbased fully convolutional networks," in Advances in neural information processing systems, 2016, pp. 379-387.

[5] S. Ren, K. He, R. Girshick, and J. Sun, "Faster R-CNN: Towards realtime object detection with region proposal networks," in Advances in neural information processing systems, 2015, pp. 91-99.

[6] L.-C. Chen, G. Papandreou, I. Kokkinos, K. Murphy, and A. L. Yuille, "Semantic image segmentation with deep convolutional nets and fully connected crfs," arXiv preprint arXiv:1412.7062, 2014.

[7] J. Long, E. Shelhamer, and T. Darrell, "Fully convolutional networks for semantic segmentation," in Proceedings of the IEEE conference on computer vision and pattern recognition, 2015, pp. 3431-3440.

[8] O. Russakovsky, J. Deng, H. Su, J. Krause, S. Satheesh, S. Ma, Z. Huang, A. Karpathy, A. Khosla, M. Bernstein et al., "Imagenet large scale visual recognition challenge," International journal of computer vision, vol. 115, no. 3, pp. 211-252, 2015.

[9] C. Zhang, S. Bengio, M. Hardt, B. Recht, and O. Vinyals, "Understanding deep learning requires rethinking generalization," arXiv preprint arXiv:1611.03530, 2016.

[10] S. Han, H. Mao, and W. J. Dally, "Deep compression: Compressing deep neural networks with pruning, trained quantization and huffman coding," arXiv preprint arXiv:1510.00149, 2015.

[11] J.-H. Luo, J. Wu, and W. Lin, "Thinet: A filter level pruning method for deep neural network compression," in Proceedings of the IEEE international conference on computer vision, 2017, pp. 5058-5066.

[12] H. Zhou, J. M. Alvarez, and F. Porikli, "Less is more: Towards compact CNNs," in European Conference on Computer Vision. Springer, 2016, pp. 662-677.

[13] W. Wen, C. Wu, Y. Wang, Y. Chen, and H. Li, "Learning structured sparsity in deep neural networks," in Advances in neural information processing systems, 2016, pp. 2074-2082.

[14] Z. Liu, J. Li, Z. Shen, G. Huang, S. Yan, and C. Zhang, "Learning efficient convolutional networks through network slimming," in Proceedings of the IEEE International Conference on Computer Vision, 2017, pp. 2736-2744.

[15] J. Wang, C. Xu, X. Yang, and J. M. Zurada, "A novel pruning algorithm for smoothing feedforward neural networks based on group lasso method," IEEE Transactions on neural networks and learning systems, vol. 29, no. 5, pp. 2012-2024, 2017.

[16] C. Louizos, M. Welling, and D. P. Kingma, "Learning sparse neural networks through $l_{-} 0$ regularization," arXiv preprint arXiv:1712.01312, 2017.

[17] Y. Shi, J. Miao, Z. Wang, P. Zhang, and L. Niu, "Feature selection with 12,1-2 regularization," IEEE Transactions on neural networks and learning systems, vol. 29, no. 10, pp. 4967-4982, 2018.

[18] X. Ding, G. Ding, J. Han, and S. Tang, "Auto-balanced filter pruning for efficient convolutional neural networks," in Thirty-Second AAAI Conference on Artificial Intelligence, 2018.

[19] Z. Huang and N. Wang, "Data-driven sparse structure selection for deep neural networks," in Proceedings of the European Conference on Computer Vision (ECCV), 2018, pp. 304-320.

[20] X. Xie, H. Zhang, J. Wang, Q. Chang, J. Wang, and N. R. Pal, "Learning optimized structure of neural networks by hidden node pruning with 11 regularization," IEEE Transactions on cybernetics, 2019. 
[21] B. N. G. Koneru and V. Vasudevan, "Sparse artificial neural networks using a novel smoothed lasso penalization," IEEE Transactions on Circuits and Systems II: Express Briefs, vol. 66, no. 5, pp. 848-852, 2019.

[22] J. Yun, P. Zheng, E. Yang, A. Lozano, and A. Aravkin, "Trimming the 11 regularizer: Statistical analysis, optimization, and applications to deep learning," in International Conference on Machine Learning, 2019, pp. 7242-7251.

[23] J. Li, Q. Qi, J. Wang, C. Ge, Y. Li, Z. Yue, and H. Sun, "OICSR: Out-InChannel Sparsity Regularization for Compact Deep Neural Networks," in Proceedings of the IEEE Conference on Computer Vision and Pattern Recognition, 2019, pp. 7046-7055.

[24] S. Lin, R. Ji, Y. Li, C. Deng, and X. Li, "Toward compact convnets via structure-sparsity regularized filter pruning," IEEE Transactions on neural networks and learning systems, 2019.

[25] H. Wang, Q. Zhang, Y. Wang, L. Yu, and H. Hu, "Structured pruning for efficient convnets via incremental regularization," in 2019 International Joint Conference on Neural Networks (IJCNN). IEEE, 2019, pp. 1-8.

[26] C. Lemaire, A. Achkar, and P.-M. Jodoin, "Structured pruning of neural networks with budget-aware regularization," in Proceedings of the IEEE Conference on Computer Vision and Pattern Recognition, 2019, pp. 9108-9116.

[27] B. O. Ayinde, T. Inanc, and J. M. Zurada, "Regularizing deep neural networks by enhancing diversity in feature extraction," IEEE Transactions on neural networks and learning systems, vol. 30, no. 9, pp. 2650-2661, 2019.

[28] S. Lin, R. Ji, C. Yan, B. Zhang, L. Cao, Q. Ye, F. Huang, and D. Doermann, "Towards optimal structured cnn pruning via generative adversarial learning," in Proceedings of the IEEE Conference on Computer Vision and Pattern Recognition, 2019, pp. 2790-2799.

[29] H. Yang, W. Wen, and H. Li, "Deephoyer: Learning sparser neural network with differentiable scale-invariant sparsity measures," arXiv preprint arXiv:1908.09979, 2019.

[30] A. Gordon, E. Eban, O. Nachum, B. Chen, H. Wu, T.-J. Yang, and E. Choi, "Morphnet: Fast \& simple resource-constrained structure learning of deep networks," in Proceedings of the IEEE Conference on Computer Vision and Pattern Recognition, 2018, pp. 1586-1595.

[31] Y. LeCun, J. S. Denker, and S. A. Solla, "Optimal brain damage," in Advances in neural information processing systems, 1990, pp. 598-605.

[32] B. Hassibi and D. G. Stork, "Second order derivatives for network pruning: Optimal brain surgeon," in Advances in neural information processing systems, 1993, pp. 164-171.

[33] D. Molchanov, A. Ashukha, and D. Vetrov, "Variational dropout sparsifies deep neural networks," in Proceedings of the 34th International Conference on Machine Learning-Volume 70. JMLR. org, 2017, pp. 2498-2507.

[34] B. Baker, O. Gupta, N. Naik, and R. Raskar, "Designing neural network architectures using reinforcement learning," arXiv preprint arXiv:1611.02167, 2016.

[35] H.-J. Kang, "Accelerator-aware pruning for convolutional neural networks," IEEE Transactions on Circuits and Systems for Video Technology, 2019.

[36] J. Frankle and M. Carbin, "The lottery ticket hypothesis: Finding sparse, trainable neural networks," arXiv preprint arXiv:1803.03635, 2018.

[37] A. Morcos, H. Yu, M. Paganini, and Y. Tian, "One ticket to win them all: generalizing lottery ticket initializations across datasets and optimizers," in Advances in Neural Information Processing Systems, 2019, pp. 4933 4943.

[38] X. Ding, X. Zhou, Y. Guo, J. Han, J. Liu et al., "Global sparse momentum sgd for pruning very deep neural networks," in Advances in Neural Information Processing Systems, 2019, pp. 6379-6391.

[39] H. Li, A. Kadav, I. Durdanovic, H. Samet, and H. P. Graf, "Pruning filters for efficient convnets," arXiv preprint arXiv:1608.08710, 2016.

[40] H. Hu, R. Peng, Y.-W. Tai, and C.-K. Tang, "Network trimming: A datadriven neuron pruning approach towards efficient deep architectures," arXiv preprint arXiv:1607.03250, 2016.

[41] Y. He, X. Zhang, and J. Sun, "Channel pruning for accelerating very deep neural networks," in Proceedings of the IEEE International Conference on Computer Vision, 2017, pp. 1389-1397.

[42] R. Yu, A. Li, C.-F. Chen, J.-H. Lai, V. I. Morariu, X. Han, M. Gao, C.-Y. Lin, and L. S. Davis, "NISP: Pruning networks using neuron importance score propagation," in Proceedings of the IEEE Conference on Computer Vision and Pattern Recognition, 2018, pp. 9194-9203.

[43] T.-W. Chin, C. Zhang, and D. Marculescu, "Layer-compensated pruning for resource-constrained convolutional neural networks," arXiv preprint arXiv:1810.00518, 2018.
[44] Y. He, J. Lin, Z. Liu, H. Wang, L.-J. Li, and S. Han, "AMC: Automl for model compression and acceleration on mobile devices," in Proceedings of the European Conference on Computer Vision (ECCV), 2018, pp. 784-800.

[45] Y. He, G. Kang, X. Dong, Y. Fu, and Y. Yang, "Soft filter pruning for accelerating deep convolutional neural networks," arXiv preprint arXiv: $1808.06866,2018$

[46] Q. Huang, K. Zhou, S. You, and U. Neumann, "Learning to prune filters in convolutional neural networks," in 2018 IEEE Winter Conference on Applications of Computer Vision (WACV). IEEE, 2018, pp. 709-718.

[47] P. Molchanov, A. Mallya, S. Tyree, I. Frosio, and J. Kautz, "Importance estimation for neural network pruning," in Proceedings of the IEEE Conference on Computer Vision and Pattern Recognition, 2019, pp. $11264-11272$.

[48] Y. He, P. Liu, Z. Wang, Z. Hu, and Y. Yang, "Filter pruning via geometric median for deep convolutional neural networks acceleration," in Proceedings of the IEEE Conference on Computer Vision and Pattern Recognition, 2019, pp. 4340-4349.

[49] C. Zhao, B. Ni, J. Zhang, Q. Zhao, W. Zhang, and Q. Tian, "Variational convolutional neural network pruning," in Proceedings of the IEEE Conference on Computer Vision and Pattern Recognition, 2019, pp. 2780-2789.

[50] Z. Liu, H. Mu, X. Zhang, Z. Guo, X. Yang, K.-T. Cheng, and J. Sun, "Metapruning: Meta learning for automatic neural network channel pruning," in Proceedings of the IEEE International Conference on Computer Vision, 2019, pp. 3296-3305.

[51] A. Krizhevsky, G. Hinton et al., "Learning multiple layers of features from tiny images," Citeseer, Tech. Rep., 2009.

[52] A. Shrivastava, A. Gupta, and R. Girshick, "Training region-based object detectors with online hard example mining," in Proceedings of the IEEE conference on computer vision and pattern recognition, 2016, pp. 761769

[53] A. Paszke, S. Gross, S. Chintala, G. Chanan, E. Yang, Z. DeVito, Z. Lin, A. Desmaison, L. Antiga, and A. Lerer, "Automatic differentiation in PyTorch," 2017.

[54] G. Huang, Y. Sun, Z. Liu, D. Sedra, and K. Q. Weinberger, "Deep networks with stochastic depth," in European conference on computer vision. Springer, 2016, pp. 646-661.

[55] M. Lin, Q. Chen, and S. Yan, "Network in network," arXiv preprint arXiv:1312.4400, 2013.

[56] K. Simonyan and A. Zisserman, "Very deep convolutional networks for large-scale image recognition," arXiv preprint arXiv:1409.1556, 2014.

[57] I. Sutskever, J. Martens, G. Dahl, and G. Hinton, "On the importance of initialization and momentum in deep learning," in International conference on machine learning, 2013, pp. 1139-1147.

[58] K. He, X. Zhang, S. Ren, and J. Sun, "Delving deep into rectifiers: Surpassing human-level performance on imagenet classification," in Proceedings of the IEEE international conference on computer vision, 2015, pp. 1026-1034.

[59] S. Lin, R. Ji, Y. Li, Y. Wu, F. Huang, and B. Zhang, "Accelerating convolutional networks via global \& dynamic filter pruning." in IJCAI, 2018, pp. 2425-2432.

[60] Z. Zhuang, M. Tan, B. Zhuang, J. Liu, Y. Guo, Q. Wu, J. Huang, and J. Zhu, "Discrimination-aware channel pruning for deep neural networks," in Advances in Neural Information Processing Systems, 2018, pp. 875-886.

[61] X. Ding, G. Ding, Y. Guo, and J. Han, "Centripetal SGD for pruning very deep convolutional networks with complicated structure," in Proceedings of the IEEE Conference on Computer Vision and Pattern Recognition, 2019, pp. 4943-4953.

[62] M. Lin, R. Ji, Y. Wang, Y. Zhang, B. Zhang, Y. Tian, and L. Shao, "HRank: Filter pruning using high-rank feature map," in Proceedings of the IEEE Conference on Computer Vision and Pattern Recognition, 2020.

[63] Y. LeCun et al., "Lenet-5, convolutional neural networks," $U R L$ : http://yann. lecun. com/exdb/lenet, vol. 20, p. 5, 2015. 